\title{
Giant planets around AF and M stars
}

\author{
Julien Rameau ${ }^{1}$, Gaël Chauvin ${ }^{1}$, Anne-Marie Lagrange ${ }^{1}$, \\ Philippe Delorme ${ }^{1}$ and Justine Lannier ${ }^{1}$ \\ ${ }^{1}$ UJF-Grenoble 1 / CNRS-INSU, Institut de Planétologie et d'Astrophysique de Grenoble \\ (IPAG) UMR 5274, Grenoble, F-38041, France \\ email: julien.rameau@obs.ujf-grenoble.fr
}

\begin{abstract}
We present the results of two three-year surveys of young and nearby stars to search for wide orbit giant planets. On the one hand, we focus on early-type and massive, namely $\beta$ Pictoris analogs. On the other hand, we observe late type and very low mass stars, i.e., M dwarfs. We report individual detections of new planetary mass objects. According to our deep detection performances, we derive the observed frequency of giant planets between these two classes of parent stars. We find frequency between 6 to $12 \%$ but we are not able to assess a/no correlation with the host-mass.
\end{abstract}

Keywords. methods: statistical, planetary systems, stars: individual (HD95086, J01033563$5515561)$

\section{Introduction}

Direct imaging is the unique viable technique to explore planetary systems beyond $10 \mathrm{AU}$ but it is, however, very challenging. The discoveries of giant planets around the young and dusty early-type stars (e.g., Marois et al. 2008, Lagrange et al. 2009) at physical separations $\leqslant 110 \mathrm{AU}$ and high-mass ratio $(q \simeq 0.002)$ strengthen a formation within the circumstellar disk either by core accretion (Pollack et al. 1996) or gravitational instability farther away (Cameron 1978). However, detailed statistical analysis of the planet properties around a large number of targets are needed to compare the relative efficiency of these formation mechanisms.

We aim at detecting substellar companions to two distinct population of stars, i.e. low mass dwarfs and early-type stars. Using statistical formalism, our goal is also to infer the influence of the host mass to the properties of the giant planet population and perhaps give an insight on their formation mechanism. Each subsample has been published in Delorme et al. (2012) and Rameau et al. (2013a).

\section{Sample, observations, and image processing}

Target selection. To optimize the detection of giant planet at short separations from their host-star, we applied several selection criteria to build two samples. Since planets are intrinsically brighter when they are young, we selected the stars to be younger than 120 Myr. We limited the volume of the sample to $50 \mathrm{pc}$ for the $\mathrm{M}$ dwarfs and $90 \mathrm{pc}$ for the AF stars to be sensitive to few AUs from the star. Our subsample thus contains more than $55 \mathrm{M}$ dwarfs among them 45 have been observed so far and 42 for the AF stars (see Fig. 1, left)

Observing strategies. The observations were done with VLT/NaCo at $\mathrm{L}^{\prime}(\lambda=3.8 \mu \mathrm{m})$ with the pupil tracking mode to enable angular differential imaging (ADI, Marois et al. 2006). The data were reduced with the IPAG-ADI pipeline (e.g. Chauvin et al. 2012). 

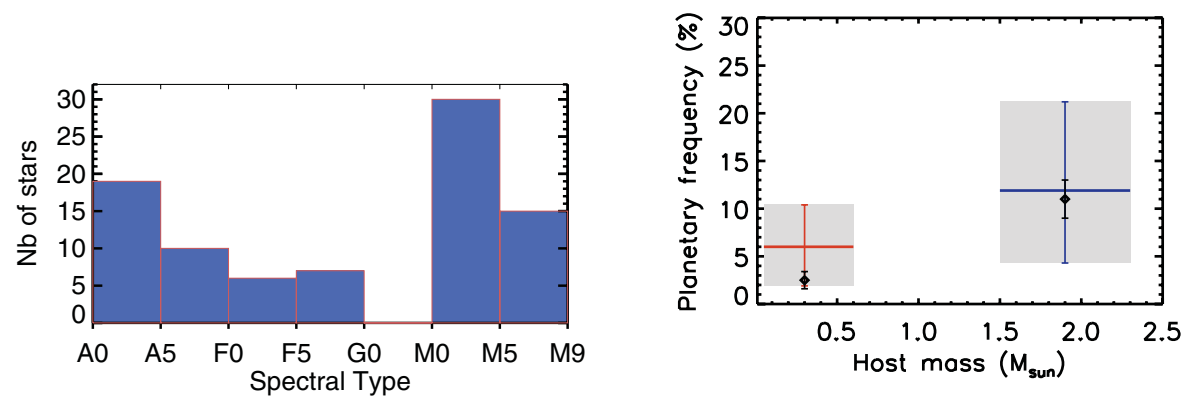

Figure 1. Left: histogram of the spectral types for our two samples. Right: Occurrence of giant planets as a function of the stellar mass in a range [1.,13] $\mathrm{M}_{\mathrm{Jup}}$ and [1.,300] AU. Black diamonds show the results for close-in giant planets from radial velocity observations (Johnson et al. 2007).

\section{Occurrence of giant planets as a function of the stellar mass}

Discoveries of new substellar companions. Delorme et al. (2013) reported the discovery of a 12-14 $\mathrm{M}_{\mathrm{Jup}}$ orbiting a pair of young (30 Myr) late M-dwarfs (2M01033563-5515561) at a projected separation of $84 \mathrm{AU}$ (system at $42 \mathrm{pc}$ ). Later on, Rameau et al. (2013b) detected a probable $4-5 \mathrm{M}_{\mathrm{Jup}}$ companion to the young $(10-17 \mathrm{Myr})$ A star HD 95086 at a projected separation of $56 \mathrm{AU}$. The last discovery lacks of good observing conditions for assessing the true detection but strong arguments tend to favor a (bound) substellar nature for the object.

Frequency of giant planets. On the one hand, for the AF sample, we typically achieved a contrast down to $10-12 \mathrm{mag}$ at 0.5 ", i.e., $1-3 \mathrm{M}_{\mathrm{Jup}}$ beyond $5-30 \mathrm{AU}$ according to the COND models (Baraffe et al. 2003). On the other hand, we reached 8.5-11 mag at 0.5 " for our $\mathrm{M}$ targets, i.e., below the jovian regime to $2 \mathrm{M}_{\mathrm{Jup}}$ beyond $4-20 \mathrm{AU}$. We then performed Monte-Carlo simulations to derive the occurrence of giant planets in a uniform grid between [1.,13] $\mathrm{M}_{\mathrm{Jup}}$ and [1.,300] AU (Rameau et al. 2013a). We found that, in our volume-limited samples, $11.9_{-7.6}^{+9.3} \%$ of AF stars harbor at least one giant planet while this rate is $6.0_{-4.1}^{+4.4} \%$ for the $\mathrm{M}$ dwarfs. The large uncertainties are due to the lack of good, i.e., below few Jupiter mass, sensitivity below 5-10 AU. In Fig.1 (right) the occurrence of giant planets is shown as a function of the stellar masses, one mass-bin corresponding to each subsample. Due to the large uncertainties, we do not assess or exclude any trend. For a comparison, we over plotted the rates of close-in giant planets for the same mass bin from Johnson et al. (2007), with the trend clearly assessed.

\section{References}

Baraffe, I., Chabrier, G., Allard, F., \& Hauschildt, P. 2003, Brown Dwarfs, 211, 41

Cameron, A. G. W. 1978, Moon and Planets, 18, 5

Chauvin, G., Lagrange, A.-M., Beust, H., et al. 2012, A\&BA, 542, A41

Delorme, P., Lagrange, A. M., Chauvin, G., et al. 2012, A\&SA, 539, A72

Delorme, P., Gagné, J., Girard, J. H., et al. 2013, A\&A, 553, L5

Johnson, J. A., Butler, R. P., Marcy, G. W., et al. 2007, APJ, 670, 833

Lagrange, A.-M., Gratadour, D., Chauvin, G., et al. 2009, A\&SA, 493, L21

Marois, C., Lafrenière, D., Doyon, R., Macintosh, B., \& Nadeau, D. 2006, APJ, 641, 556

Marois, C., Macintosh, B., Barman, T., et al. 2008, Science, 322, 1348

Pollack, J. B., Hubickyj, O., Bodenheimer, P., et al. 1996, Icarus, 124, 62

Rameau, J., Chauvin, G., Lagrange, A.-M., et al. 2013, A\&A, 553, A60

Rameau, J., Chauvin, G., Lagrange, A.-M., et al. 2013, arXiv:1305.7428 